\title{
On Exponential Stabilizability for a Class of Switched Nonlinear Systems with Mixed Time-Varying Delays
}

\author{
Jie $Q i^{1,2}$ and Yuangong Sun ${ }^{1}$ \\ ${ }^{1}$ School of Mathematics, University of Jinan, Jinan, Shandong 250022, China \\ ${ }^{2}$ School of Automation and Electrical Engineering, University of Jinan, Jinan, Shandong 250022, China \\ Correspondence should be addressed to Yuangong Sun; sunyuangong@163.com
}

Received 9 June 2014; Revised 2 September 2014; Accepted 2 September 2014; Published 4 December 2014

Academic Editor: Hamid R. Karimi

Copyright (c) 2014 J. Qi and Y. Sun. This is an open access article distributed under the Creative Commons Attribution License, which permits unrestricted use, distribution, and reproduction in any medium, provided the original work is properly cited.

We study the exponential stabilizability for a class of switched nonlinear systems with mixed time-varying delays. By using a new technique developed for positive systems, we design the average dwell time switching under which the switched nonlinear system is exponentially stable for any bounded delays. Finally, numerical examples are worked out to illustrate the main theoretical result.

\section{Introduction}

A switched system which consists of a series of dynamical subsystems and a switching signal is a type of hybrid dynamical systems. Switched systems can be used to model many phenomena which cannot be described by purely continuous or purely discrete processes. Due to its broad applications in traffic control, chemical processing, switching power converters, and network control, the theory of switched systems has historically a position of great importance in systems theory and has been studied extensively in recent years [15].

Up to now, the stability of switched systems has attracted many researchers' attention. For stability issues, two main problems have been investigated in the literature. One is to find conditions that guarantee asymptotic stability of the switched system under arbitrary switching. For this case, the common Lyapunov function is required for all subsystems $[6,7]$. The other is to identify those switching signals for which the switched system is asymptotically stable, that is, stability of switched systems under constrained switching. For this case, the multiple-Lyapunov functions are a powerful and effective tool, and average dwell time (ADT) approaches have been used to investigate the stability and stabilization problems in [8].

Recently, positive switched system receives much attention. In the theory of positive switched systems, the stability problem is investigated extensively by many researchers [913], especially for the stability under arbitrary switching. It is well known that a common linear copositive Lyapunov function (CLCLF) is usually applied to the asymptotic stability of positive switched systems under arbitrary switching. Recently, necessary and sufficient conditions for the existence of CLCLFs were established in $[13,14]$. For the case when the positive switched system does not share a CLCLF, a multiple linear copositive Lyapunov functional method was used in [15]. Some other methods to stability of switched nonlinear systems were proposed in [16-21].

In this paper, we study the exponential stabilizability for a class of switched nonlinear systems with mixed timevarying delays. Due to the the existence of both discrete and distributed time-varying delays and the assumption that the system is not necessarily positive, a new technique developed for positive systems is employed to the exponential stability under ADT switching for a class of switched nonlinear systems with mixed time-varying delays.

Notation. Throughout this paper, $\langle m\rangle$ is the set of integers $\{1,2, \ldots, m\}$ for any positive integer $m$. Say a real vector $x>$ $0(<0)$ if all entries of $x$ are positive (negative). The norm of the vector $x \in R^{n}$ is defined to be $\|x\|=\max _{i=1,2, \ldots, n}\left\{\left|x_{i}\right|\right\}$. Say a square matrix is Metzler if its off-diagonal entries are nonnegative. Say a matrix is nonnegative if all its entries are nonnegative. 


\section{Problem Statements and Preliminaries}

Consider the following switched nonlinear systems with mixed time-varying delays:

$$
\begin{aligned}
\dot{x}(t)= & A_{\sigma(t)} f(x(t))+B_{\sigma(t)} f\left(x\left(t-d_{1}(t)\right)\right) \\
& +C_{\sigma(t)} \int_{-d_{2}(t)}^{0} f(x(t+s)) d s, \quad t \geq 0,
\end{aligned}
$$

where $x(t)=\left[x_{1}(t), x_{2}(t), \ldots, x_{n}(t)\right]^{T} \in R^{n} ; d_{1}(t)$ and $d_{2}(t)$ are piecewise continuous time-delays satisfying $0<d_{1}(t) \leq$ $\tau_{1}, 0<d_{2}(t) \leq \tau_{2}$, and $\tau_{1}>0$ and $\tau_{2}>0$ are constants; $\sigma$ is a piecewise constant function of time, called a switching signal, that takes its values in the finite set $\langle m\rangle=\{1, \ldots, m\} ; A_{p}=$ $\left[a_{i j}^{(p)}\right], B_{p}=\left[b_{i j}^{(p)}\right], C_{p}=\left[c_{i j}^{(p)}\right] \in R^{n \times n}, p \in\langle m\rangle$, are constant matrices; $f(x)=\left[x_{1}^{\gamma_{1}}, \ldots, x_{n}^{\gamma_{n}}\right]^{T}$ with $0<\gamma_{i}=$ odd $/$ odd $\leq 1$ for $i \in\langle n\rangle$.

For the particular case when $\gamma_{i}=1$ and $C_{p} \equiv 0$, system (1) reduces to the following switched linear delay system:

$$
\dot{x}(t)=A_{\sigma(t)} x(t)+B_{\sigma(t)} x\left(t-d_{1}(t)\right), \quad t \geq 0 .
$$

Under the assumption that $A_{p}$ is Metzler and $B_{p}$ is nonnegative for $p \in\langle m\rangle$, it was proved in [11] that system (2) is asymptotically stable under arbitrary switching if there exists a common vector $\xi=\left[\xi_{1}, \xi_{2}, \ldots, \xi_{n}\right]^{T}>0$ such that

$$
\left[A_{p}+B_{p}\right] \xi \prec 0, \quad p \in\langle m\rangle \text {. }
$$

If we do not assume that $A_{p}$ is Metzler and $B_{p}$ and $C_{p}$ are nonnegative for $p \in\langle m\rangle$, set $\bar{A}_{p}=\left[\bar{a}_{i j}^{(p)}\right]$ with $\bar{a}_{i i}^{(p)}=a_{i i}^{(p)}$ and $\bar{a}_{i j}^{(p)}=\left|a_{i j}^{(p)}\right|$ for $i \neq j, \bar{B}_{p}=\left[\bar{b}_{i j}^{(p)}\right]$ with $\bar{b}_{i j}^{(p)}=\left|b_{i j}^{(p)}\right|$, and $\bar{C}_{p}=\left[\bar{c}_{i j}^{(p)}\right]$ with $\bar{c}_{i j}^{(p)}=\left|c_{i j}^{(p)}\right|$. It was proved in [22] that system (2) is asymptotically stable under arbitrary switching if there exists a common vector $\xi=\left[\xi_{1}, \xi_{2}, \ldots, \xi_{n}\right]^{T}>0$ such that

$$
\left[\bar{A}_{p}+\bar{B}_{p}\right] \xi \prec 0, \quad p \in\langle m\rangle \text {. }
$$

However, in many cases, condition (3) or (4) may not hold. It may be natural to assume that there exist a set of vectors $\xi^{(p)}=\left[\xi_{1}^{(p)}, \xi_{2}^{(p)}, \ldots, \xi_{n}^{(p)}\right]^{T}>0$ such that

$$
\left[\bar{A}_{p}+\bar{B}_{p}\right] \xi^{(p)} \prec 0, \quad p \in\langle m\rangle \text {. }
$$

In this paper, we will study the exponential stability of system (1) under the milder condition of form (5).

Let $C\left([-\tau, 0], R^{n}\right)$ be the Banach space of all continuous functions on $[-\tau, 0]$ with values in $R^{n}$ normed by the maximum norm $\|\phi\|_{\tau}=\max _{\theta \in[-\tau, 0]}\|\phi(\theta)\|$, where $\phi=$ $\left[\phi_{1}, \phi_{2}, \ldots, \phi_{n}\right]^{T}$ and $\tau=\max \left\{\tau_{1}, \tau_{2}\right\}$.

For a switching signal $\sigma(t)$ and any $t_{2}>t_{1} \geq 0$, let $N_{\sigma}\left(t_{1}, t_{2}\right)$ denote the number of discontinuities of $\sigma(t)$ in the open interval $\left(t_{1}, t_{2}\right)$. We say that $\sigma(t)$ has an $\operatorname{ADT} \tau_{a}>0$ if $\tau_{a}$ satisfies

$$
N_{\sigma}\left(t_{1}, t_{2}\right) \leq \frac{t_{2}-t_{1}}{\tau_{a}} .
$$

Throughout this paper, system (1) is said to be exponentially stabilizable via ADT switching, if for any initial function $\phi \in C\left([-\tau, 0], R^{n}\right)$ there exist positive constants $K>0, \beta>0$, and $\tau_{a}>0$ (which are usually relative to the given initial function $\phi$ ) such that the corresponding solution $x(t)$ of system (1) under any switching with ADT $\tau_{a}$ satisfies $\|x(t)\| \leq K e^{-\beta t}$ for $t \geq 0$.

\section{Main Results}

In the sequel, we assume that there exist vectors $\xi^{(p)}>0, p \in$ $S$, such that

$$
\left[\bar{A}_{p}+\bar{B}_{p}+\tau_{2} \bar{C}_{p}\right] \xi^{(p)} \prec 0, \quad p \in\langle m\rangle
$$

Then, we have the following global exponential stability criterion for system (1).

Theorem 1. System (1) is exponentially stabilizable via ADT switching if there exist vectors $\xi^{(p)}>0, p \in S$, such that (7) holds.

Proof. For a given switching sequence $0=t_{0}<t_{1}<\cdots<$ $t_{k}<t_{k+1}<\cdots$, let $\sigma(t)=i_{k} \in S$ for $t \in\left[t_{k}, t_{k+1}\right)$; that is, the $i_{k}$ th subsystem is active on $\left[t_{k}, t_{k+1}\right)$. For any given $\phi \in$ $C\left([-\tau, 0], R^{n}\right)$, we choose an appropriate constant $1 \geq \rho>0$ such that $\rho^{1 / \gamma_{i}}\|\phi\|_{\tau} \leq 1$ for $i \in\langle n\rangle$. Set $y_{i}(t)=\rho^{1 / \gamma_{i}} x(t)$ for $i \in\langle n\rangle$. Then, we have

$$
\begin{aligned}
\dot{y}_{i}(t)=\rho^{\left(1 / \gamma_{i}\right)-1} \sum_{j=1}^{n}\left[a_{i j} y_{j}^{\gamma_{j}}(t)+b_{i j} y_{j}^{\gamma_{j}}\left(t-d_{1}(t)\right)\right. & \\
& \left.+\int_{-d_{2}(t)}^{0} c_{i j} y_{j}^{\gamma_{j}}(t+s) d s\right], \quad i \in\langle n\rangle .
\end{aligned}
$$

Denote $\eta=\min _{i \in\langle n\rangle} \min _{p \in\langle m\rangle}\left\{\xi_{i}^{(p)}\right\}$. Noting that

$$
\lim _{\lambda \rightarrow 0^{+}} e^{\lambda \tau_{1}}=1, \quad \lim _{\lambda \rightarrow 0^{+}} \frac{e^{\lambda \tau_{2}}-1}{\lambda}=\tau_{2}
$$

we can get from (7) that there exists an appropriate constant $\lambda>0$ such that

$$
\begin{array}{r}
\frac{1}{\eta} \rho^{1 / \gamma_{i}-1} \sum_{j=1}^{n}\left[\bar{a}_{i j}^{(p)}+e^{\lambda \tau_{1}} \bar{b}_{i j}^{(p)}+\frac{e^{\lambda \tau_{2}}-1}{\lambda} \bar{c}_{i j}^{(p)}\right] \xi_{j}^{(p)} \\
+\frac{\lambda}{\gamma_{i}}\left[\frac{\xi_{i}^{(p)}}{\eta}\right]^{1 / \gamma_{i}} \prec 0, \quad i \in\langle n\rangle, p \in\langle m\rangle .
\end{array}
$$


Let $z_{i}(t)=e^{\left(\lambda / \gamma_{i}\right) t} y_{i}(t)$. Then, system (1) reduces to the following system:

$$
\begin{aligned}
\dot{z}_{i}(t)=\frac{\lambda}{\gamma_{i}} z_{i}(t) & +\rho^{1 / \gamma_{i}-1} e^{\lambda\left(1 / \gamma_{i}-1\right) t} \\
\times \sum_{j=1}^{n}\left[a_{i j} z_{j}^{\gamma_{j}}(t)+b_{i j} e^{\lambda d_{1}(t)} z_{j}^{\gamma_{j}}\left(t-d_{1}(t)\right)\right. & \\
& \left.\quad \int_{-d_{2}(t)}^{0} c_{i j} e^{-\lambda s} z_{j}^{\gamma_{j}}(t+s) d s\right],
\end{aligned}
$$

where $t \geq 0$ and $i \in\langle n\rangle$.

First, we get from (10) that there exists a constant $\zeta>1$ such that, for any $i \in\langle n\rangle, p \in\langle m\rangle$, and $\delta \in(1, \zeta)$,

$$
\begin{gathered}
\frac{\delta}{\eta} \rho^{1 / \gamma_{i}-1} \sum_{j=1}^{n}\left[\bar{a}_{i j}^{(p)}+e^{\lambda \tau_{1}} \bar{b}_{i j}^{(p)}+\frac{e^{\lambda \tau_{2}}-1}{\lambda} \bar{c}_{i j}^{(p)}\right] \xi_{j}^{(p)} \\
+\frac{\lambda}{\gamma_{i}}\left[\frac{\delta \xi_{i}^{(p)}}{\eta}\right]^{1 / \gamma_{i}} \prec 0 .
\end{gathered}
$$

Set $\|z\|_{\tau}=\max _{\theta \in[-\tau, 0]}\|z(\theta)\|$. Then, we have that, for any $\delta \epsilon$ $(1, \zeta)$ and $i \in\langle n\rangle$,

$$
\begin{aligned}
\left|z_{i}(0)\right| & =\left|y_{i}(0)\right| \\
& =\rho^{1 / \gamma_{i}} x_{i}(0) \leq \rho^{1 / \gamma_{i}}\|\phi\|_{\tau} \leq 1<\left[\delta \frac{\xi_{i}^{\left(i_{0}\right)}}{\eta}\right]^{1 / \gamma_{i}} .
\end{aligned}
$$

By the continuity of $z_{i}(t)$ at $t=0$, there exists $t^{*}: t_{0}<t^{*}<t_{1}$ such that

$$
\left|z_{i}(t)\right|<\left[\delta \frac{\xi_{i}^{\left(i_{0}\right)}}{\eta}\right]^{1 / \gamma_{i}}, \quad t \in\left[t_{0}, t^{*}\right], i \in\langle n\rangle .
$$

We now prove that (14) holds on $\left[t_{0}, t_{1}\right)$ for any given $\delta \in$ $(1, \zeta)$. Otherwise, there exist $\widetilde{t}: t^{*}<\widetilde{t}<t_{1}$ and at least one index $k_{0} \in\langle n\rangle$ such that, for $t \in\left[t_{0}, \widetilde{t}\right)$ and $i \in\langle n\rangle$,

$$
\left|z_{k_{0}}(\tilde{t})\right|=\left[\delta \frac{\xi_{k_{0}}^{\left(i_{0}\right)}}{\eta}\right]^{1 / \gamma_{i}}, \quad\left|z_{i}(t)\right|<\left[\delta \frac{\xi_{i}^{\left(i_{0}\right)}}{\eta}\right]^{1 / \gamma_{i}} .
$$

It implies that $D_{-}\left|z_{k_{0}}(\widetilde{t})\right| \geq 0$. On the other hand, for $t \in\left[t_{0}, \widetilde{t}\right)$ and $z_{k_{0}}(t) \neq 0$, we get from (11) that

$$
\begin{aligned}
D_{-}\left|z_{k_{0}}(t)\right|= & \dot{z}_{k_{0}}(t) \operatorname{sign} z_{k_{0}}(t) \\
\leq & \frac{\lambda}{\gamma_{k_{0}}}\left|z_{k_{0}}(t)\right|+\rho^{1 / \gamma_{k_{0}}-1} e^{\lambda\left(1 / \gamma_{k_{0}}-1\right) t} \\
& \quad \times \sum_{j=1}^{n}\left[\bar{a}_{k_{0} j}^{\left(i_{0}\right)}\left|z_{j}(t)\right|^{\gamma_{j}}+\bar{b}_{k_{0} j}^{\left(i_{0}\right)} e^{\lambda \tau_{1}}\left|z_{j}\left(t-d_{1}(t)\right)\right|^{\gamma_{j}}\right. \\
& \left.\quad+\int_{-d_{2}(t)}^{0} \bar{c}_{k_{0} j}^{\left(i_{0}\right)} e^{-\lambda s}\left|z_{j}(t+s)\right|^{\gamma_{j}} d s\right] .
\end{aligned}
$$

Therefore, by (15), we have that

$$
\begin{aligned}
& D_{-}\left|z_{k_{0}}(\tilde{t})\right| \\
& \leq \frac{\lambda}{\gamma_{k_{0}}}\left[\delta \frac{\xi_{k_{0}}^{\left(i_{0}\right)}}{\eta}\right]^{1 / \gamma_{k_{0}}}+\frac{\delta}{\eta} \rho^{1 / \gamma_{k_{0}}-1} e^{\lambda\left(1 / \gamma_{k_{0}}-1\right) t} \\
& \quad \times \sum_{j=1}^{n}\left[\bar{a}_{k_{0} j}^{\left(i_{0}\right)}+e^{\lambda \tau_{1}} \bar{b}_{k_{0} j}^{\left(i_{0}\right)}+\bar{c}_{k_{0} j}^{\left(i_{0}\right)} \int_{-\tau_{2}}^{0} e^{-\lambda s} d s\right] \xi_{j}^{\left(i_{0}\right)} \\
& =\frac{\delta}{\eta} \rho^{1 / \gamma_{k_{0}}-1} e^{\lambda\left(1 / \gamma_{k_{0}}-1\right) t} \\
& \quad \times \sum_{j=1}^{n}\left[\bar{a}_{k_{0} j}^{\left(i_{0}\right)}+e^{\lambda \tau_{1}} \bar{b}_{k_{0} j}^{\left(i_{0}\right)}+\frac{e^{\lambda \tau_{2}}-1}{\lambda} \bar{c}_{k_{0} j}^{\left(i_{0}\right)}\right] \xi_{j}^{\left(i_{0}\right)} \\
& \quad+\frac{\lambda}{\gamma_{k_{0}}}\left[\delta \frac{\xi_{k_{0}}^{\left(i_{0}\right)}}{\eta}\right]^{1 / \gamma_{k_{0}}} .
\end{aligned}
$$

From (12), we have that

$$
\sum_{j=1}^{n}\left(\bar{a}_{k_{0} j}^{\left(i_{0}\right)}+e^{\lambda \tau_{1}} \bar{b}_{k_{0} j}^{\left(i_{0}\right)}+\frac{e^{\lambda \tau_{2}}-1}{\lambda} \bar{c}_{k_{0} j}^{\left(i_{0}\right)}\right) \xi_{j}^{\left(i_{0}\right)}<0 .
$$

Noting that $0<\gamma_{k_{0}} \leq 1$, we get from (17) and (18) that

$$
\begin{aligned}
D_{-}\left|z_{k_{0}}(\widetilde{t})\right| \leq & \frac{\delta}{\eta} \rho^{1 / \gamma_{k_{0}}-1} \\
& \times \sum_{j=1}^{n}\left[\bar{a}_{k_{0} j}^{\left(i_{0}\right)}+e^{\lambda \tau_{1}} \bar{b}_{k_{0} j}^{\left(i_{0}\right)}+\frac{e^{\lambda \tau_{2}}-1}{\lambda} \bar{c}_{k_{0} j}^{\left(i_{0}\right)}\right) \xi_{j}^{\left(i_{0}\right)} \\
& +\frac{\lambda}{\gamma_{k_{0}}}\left[\delta \frac{\xi_{k_{0}}^{\left(i_{0}\right)}}{\eta}\right]^{1 / \gamma_{k_{0}}} .
\end{aligned}
$$

It yields that $D_{-}\left|y_{k_{0}}(\widetilde{t})\right|<0$ from (12) with $p=i_{0}$ and $i=k_{0}$, which contradicts the fact that $D_{-}\left|z_{k_{0}}(\widetilde{t})\right| \geq 0$. Therefore, (14) holds on $\left[t_{0}, t_{1}\right)$ for any $\delta \in(1, \zeta)$. By letting $\delta$ and $t$ tend to 1 and $t_{1}$, respectively, we have that

$$
\begin{aligned}
\left|z_{i}\left(t_{1}\right)\right| & \leq\left[\frac{\xi_{i}^{\left(i_{0}\right)}}{\eta}\right]^{1 / \gamma_{i}} \\
& =\left[\frac{\xi_{i}^{\left(i_{0}\right)} \xi_{i}^{\left(i_{1}\right)}}{\xi_{i}^{\left(i_{1}\right)} \eta}\right]^{1 / \gamma_{i}} \leq\left[\mu \frac{\xi_{i}^{\left(i_{1}\right)}}{\eta}\right]^{1 / \gamma_{i}}, \quad i \in\langle n\rangle,
\end{aligned}
$$

where $\mu=\max \left\{\xi_{i}^{(p)} / \xi_{i}^{(q)}: p, q \in\langle m\rangle, i \in\langle n\rangle\right\}$. Therefore,

$$
\left|z_{i}\left(t_{1}\right)\right|<\left[\mu \delta \frac{\xi_{i}^{\left(i_{1}\right)}}{\eta}\right]^{1 / \gamma_{i}}, \quad \forall \delta \in(1, \zeta) .
$$


Since $y_{i}(t)$ is continuous at $t=t_{1}$, by repeating the above procedure, we can conclude that

$$
\left|z_{i}(t)\right| \leq\left[\mu \frac{\xi_{i}^{\left(i_{1}\right)}}{\eta}\right]^{1 / \gamma_{i}}, \quad t \in\left[t_{1}, t_{2}\right), i \in\langle n\rangle .
$$

By induction, we have that, for each $k \in\{0,1,2, \ldots\}$,

$$
\left|z_{i}(t)\right| \leq\left[\mu^{k} \frac{\xi_{i}^{\left(i_{k}\right)}}{\eta}\right]^{1 / \gamma_{i}}, \quad t \in\left[t_{k}, t_{k+1}\right), i \in\langle n\rangle .
$$

Therefore, for any $t \in\left[t_{k}, t_{k+1}\right)$, we get from (23) that

$$
\begin{aligned}
\left|x_{i}(t)\right| & =\rho^{-1 / \gamma_{i}} e^{-\left(\lambda / \gamma_{i}\right) t}\left|z_{i}(t)\right| \\
& \leq \rho^{-1 / \gamma_{i}} e^{-\left(\lambda / \gamma_{i}\right) t}\left[\mu^{k} \frac{\xi_{i}^{\left(i_{k}\right)}}{\eta}\right]^{1 / \gamma_{i}} \\
& \leq \rho^{-1 / \gamma_{i}} e^{-\left(\lambda / \gamma_{i}\right) t}\left[\mu^{t / \tau_{a}} \mu\right]^{1 / \gamma_{i}} \\
& =\rho^{-1 / \gamma_{i}} e^{-\left(\lambda / \gamma_{i}\right) t}\left[\mu e^{\left(\ln \mu / \tau_{a}\right) t}\right]^{1 / \gamma_{i}} \\
& =\rho^{-1 / \gamma_{i}} \mu^{1 / \gamma_{i}}\left[e^{-\left(\lambda-\ln \mu / \tau_{a}\right) t}\right]^{1 / \gamma_{i}} .
\end{aligned}
$$

If we set $K=\rho^{-1 / \gamma_{\min }} \mu^{1 / \gamma_{\min }}$ and $\beta=\left(\lambda-\ln \mu / \tau_{a}\right)\left(1 / \gamma_{\max }\right)$, where $\gamma_{\min }=\min _{i \in\langle n\rangle} \gamma_{i}, \gamma_{\max }=\max _{i \in\langle n\rangle} \gamma_{i}$ and $\tau_{a}$ satisfies $\tau_{a}>\ln \mu / \lambda$. By (24), we have that system (1) is exponentially stabilizable via ADT switching. This completes the proof of Theorem 1.

If there exists a common vector $\xi>0$ such that

$$
\left[\bar{A}_{p}+\bar{B}_{p}+\tau_{2} \bar{C}_{p}\right] \xi \prec 0, \quad p \in\langle m\rangle
$$

we have that $\mu=1$. Then, Theorem 1 yields the following corollary.

Corollary 2. If there exists a common vector $\xi>0$ such that (25) holds, then system (1) is globally exponentially stable under any switching.

\section{Illustrative Examples}

Example 1. Consider system (1) with $0<d_{1}(t) \leq 0.1,0<$ $d_{2}(t) \leq 0.2, \gamma_{1}=7 / 9, \gamma_{2}=1$, and

$$
\begin{array}{ll}
A_{1}=\left[\begin{array}{cc}
-1 & -0.1 \\
0.05 & -0.5
\end{array}\right], & A_{2}=\left[\begin{array}{cc}
-0.6 & 0.2 \\
-0.1 & -1
\end{array}\right], \\
B_{1}=\left[\begin{array}{cc}
0.1 & -0.1 \\
0 & 0
\end{array}\right], & B_{2}=\left[\begin{array}{cc}
0 & 0 \\
0.1 & -0.05
\end{array}\right], \\
C_{1}=\left[\begin{array}{ll}
-0.5 & 0 \\
1.25 & 1
\end{array}\right], & C_{2}=\left[\begin{array}{ll}
0.75 & 1.25 \\
-0.5 & 0.25
\end{array}\right] .
\end{array}
$$

Based on a straightforward computation, we have that

$$
\begin{aligned}
& \bar{A}_{1}+\bar{B}_{1}+0.2 \cdot \bar{C}_{1} \triangleq \Phi_{1}=\left[\begin{array}{cc}
-0.8 & 0.2 \\
0.3 & -0.3
\end{array}\right], \\
& \bar{A}_{2}+\bar{B}_{2}+0.2 \cdot \bar{C}_{2} \triangleq \Phi_{2}=\left[\begin{array}{cc}
-0.45 & 0.45 \\
0.3 & -0.9
\end{array}\right] .
\end{aligned}
$$

We see that there does not exist a vector $\xi=\left[\xi_{1}, \xi_{2}\right]^{T}>0$ such that $\Phi_{p} \xi<0$ for $p=1,2$. Otherwise, we can get $\xi_{1}<\xi_{2}$ and $\xi_{2}<\xi_{1}$. This is a contradiction. Therefore, condition (25) is invalid for this case. However, there exist two vectors $\xi^{(1)}=$ $[0.25,0.5]^{T}$ and $\xi^{(2)}=[0.5,0.29]^{T}$ such that

$$
\Phi_{p} \xi^{(p)} \prec 0, \quad p=1,2
$$

that is, condition (7) holds. For any initial condition $\phi$ satisfying $\|\phi\|_{\tau} \leq 1$, we can choose $\rho=1$. It is not difficult to verify that (10) holds for $\lambda=0.12$ and $\rho=1$. Therefore, by Theorem 1, all solutions satisfying the initial condition $\|\phi\|_{\tau} \leq 1$ are exponentially stabilizable via the switching with $\mathrm{ADT} \tau_{a}>\ln \mu / \lambda \approx 5.78$.

Example 2. Consider system (1) with $0<d_{1}(t) \leq 0.5, \gamma_{1}=$ $\gamma_{2}=\gamma_{3}=1$, and

$$
\begin{gathered}
A_{1}=\left[\begin{array}{ccc}
-3 & 0.3 & 0.2 \\
0.4 & -1.2 & 0.2 \\
0.2 & 0 & -2.5
\end{array}\right], \quad A_{2}=\left[\begin{array}{ccc}
-1.5 & 0.3 & 0.1 \\
0.2 & -3 & 0.4 \\
0.1 & 0.5 & -3
\end{array}\right], \\
B_{1}=\left[\begin{array}{ccc}
1 & -0.2 & 0.3 \\
0.6 & -0.2 & 0 \\
-0.3 & 0.5 & 0.5
\end{array}\right], \quad B_{2}=\left[\begin{array}{ccc}
-0.5 & 0.7 & 0 \\
0.1 & 0.5 & -0.2 \\
0.2 & -0.1 & 1
\end{array}\right], \\
C_{1}=C_{2}=C_{3}=0 .
\end{gathered}
$$

For this case, we have that

$$
\begin{gathered}
\bar{A}_{1}+\bar{A}_{2} \triangleq \Psi_{1}=\left[\begin{array}{ccc}
-2 & 0.5 & 0.5 \\
1 & -1 & 0.2 \\
0.5 & 0.5 & -2
\end{array}\right], \\
\bar{A}_{2}+\bar{B}_{2} \triangleq \Psi_{2}=\left[\begin{array}{ccc}
-1 & 1 & 0.1 \\
0.3 & -2.5 & 0.6 \\
0.3 & 0.6 & -2
\end{array}\right] .
\end{gathered}
$$

It is not difficult to verify that there does not exist a vector $\xi=\left[\xi_{1}, \xi_{2}, \xi_{3}\right]^{T}>0$ such that $\Psi_{p} \xi<0$ for $p=1,2$. Note that (10) reduces to

$$
\sum_{j=1}^{n}\left[\bar{a}_{i j}^{(p)}+e^{\lambda \tau_{1}} \bar{b}_{i j}^{(p)}\right] \xi_{j}^{(p)}+\lambda \xi_{i}^{(p)} \prec 0, \quad i=1,2,3, p=1,2 .
$$

By solving the above inequality, we get a solution $\xi^{(1)}=$ $[1,2.1,1]^{T}, \xi^{(2)}=[2.1,1,1]^{T}$, and $\lambda=0.3$. Therefore, by Theorem 1 and its proof, we have that system (1) is exponentially stabilizable via the switching with ADT $\tau_{a}>$ $\ln \mu / \lambda \approx 2.4731$.

\section{Conclusion}

This paper has investigated the exponential stabilizability for a class of switched nonlinear systems with mixed timevarying delays by using a new technique developed for positive systems. By using a new method developed for positive 
systems, we design the appropriate ADT switching under which the system is exponentially stable. The main results generalize some existing results in the literature. Two numerical examples are also worked out to illustrate the effectiveness and sharpness of the given theoretical result. Stability analysis for the more general switched nonlinear systems with mixed time delays will be further investigated in the future.

\section{Conflict of Interests}

The authors declare that there is no conflict of interests regarding the publication of this paper.

\section{Acknowledgments}

The authors thank the reviewers for their valuable comments on this paper. This work was supported by the Natural Science Foundation of Shandong Province under Grant no. JQ201119 and the National Natural Science Foundation of China under Grant nos. 61174217, 61374074, and 61473133.

\section{References}

[1] D. Liberzon, Switching in Systems and Control, Birkhäuser, Boston, Mass, USA, 2003.

[2] G. Xie and L. Wang, "Periodic stabilizability of switched linear control systems," Automatica, vol. 45, no. 9, pp. 2141-2148, 2009.

[3] B. Du and X. Zhang, "Delay-dependent stability analysis and synthesis for uncertain impulsive switched system with mixed delays," Discrete Dynamics in Nature and Society, vol. 2011, Article ID 381571, 9 pages, 2011.

[4] M. De la Sen and A. Ibeas, "Stability results of a class of hybrid systems under switched continuous-time and discretetime control," Discrete Dynamics in Nature and Society, vol. 2009, Article ID 315713, 28 pages, 2009.

[5] R. Guo, "Stability analysis of a class of switched nonlinear systems with an improved average dwell time method," Abstract and Applied Analysis, vol. 2014, Article ID 214756, 8 pages, 2014.

[6] R. Shorten, K. S. Narendra, and O. Mason, "A result on common quadratic Lyapunov functions," IEEE Transactions on Automatic Control, vol. 48, no. 1, pp. 110-113, 2003.

[7] A. Ibeas and M. de la Sen, "Exponential stability of simultaneously triangularizable switched systems with explicit calculation of a common Lyapunov function," Applied Mathematics Letters, vol. 22, no. 10, pp. 1549-1555, 2009.

[8] M. S. Branicky, "Multiple Lyapunov functions and other analysis tools for switched and hybrid systems," IEEE Transactions on Automatic Control, vol. 43, no. 4, pp. 475-482, 1998.

[9] L. Gurvits, R. Shorten, and O. Mason, "On the stability of switched positive linear systems," IEEE Transactions on Automatic Control, vol. 52, no. 6, pp. 1099-1103, 2007.

[10] L. Fainshil, M. Margaliot, and P. Chigansky, "On the stability of positive linear switched systems under arbitrary switching laws," IEEE Transactions on Automatic Control, vol. 54, no. 4, pp. 897-899, 2009.

[11] X. Liu and C. Dang, "Stability analysis of positive switched linear systems with delays," IEEE Transactions on Automatic Control, vol. 56, no. 7, pp. 1684-1690, 2011.
[12] J. Qi and Y. Sun, "Global exponential stability of certain switched systems with time-varying delays," Applied Mathematics Letters, vol. 26, no. 7, pp. 760-765, 2013.

[13] E. Fornasini and M. E. Valcher, "Linear copositive Lyapunov functions for continuous-time positive switched systems," IEEE Transactions on Automatic Control, vol. 55, no. 8, pp. 1933-1937, 2010.

[14] Z. Wu and Y. Sun, "On easily verifiable conditions for the existence of common linear copositive Lyapunov functions," IEEE Transactions on Automatic Control, vol. 58, no. 7, pp. 18621865, 2013.

[15] X. Zhao, L. Zhang, P. Shi, and M. Liu, "Stability of switched positive linear systems with average dwell time switching," Automatica, vol. 48, no. 6, pp. 1132-1137, 2012.

[16] C. Qin, Z. R. Xiang, and H. R. Karimi, "Robust $H_{\infty}$ reliable control of delta operator switched systems with time-varying delays under asynchronous switching," Transactions of the Institute of Measurement and Control, 2014.

[17] Z. Wu, M. Cui, P. Shi, and H. R. Karimi, "Stability of stochastic nonlinear systems with state-dependent switching," IEEE Transactions on Automatic Control, vol. 58, no. 8, pp. 1904-1918, 2013.

[18] Y. Kao, C. Wang, H. R. Karimi, and R. Bi, "Global stability of coupled Markovian switching reaction-diffusion systems on networks," Nonlinear Analysis: Hybrid Systems, vol. 13, pp. 61-73, 2014.

[19] H. Zhang and J. Wang, "Combined feedback-feedforward tracking control for networked control systems with probabilistic delays," Journal of the Franklin Institute: Engineering and Applied Mathematics, vol. 351, no. 6, pp. 3477-3489, 2014.

[20] H. Zhang, Y. Shi, and J. Wang, "On energy-to-peak filtering for nonuniformly sampled nonlinear systems: a markovian jump system approach," IEEE Transactions on Fuzzy Systems, vol. 22, no. 1, pp. 212-222, 2014.

[21] H. Zhang, Y. Shi, and J. Wang, “Observer-based tracking controller design for networked predictive control systems with uncertain Markov delays," International Journal of Control, vol. 86, no. 10, pp. 1824-1836, 2013.

[22] Y. Sun, "Delay-independent stability of switched linear systems with unbounded time-varying delays," Abstract and Applied Analysis, vol. 2012, Article ID 560897, 11 pages, 2012. 


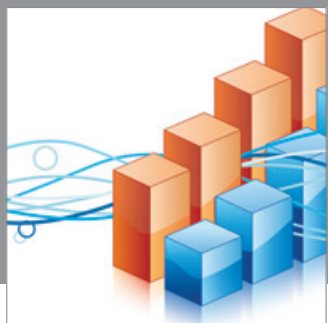

Advances in

Operations Research

mansans

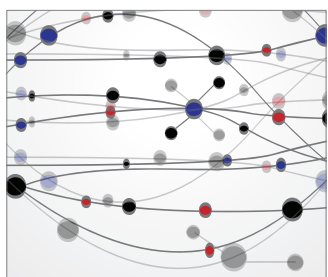

The Scientific World Journal
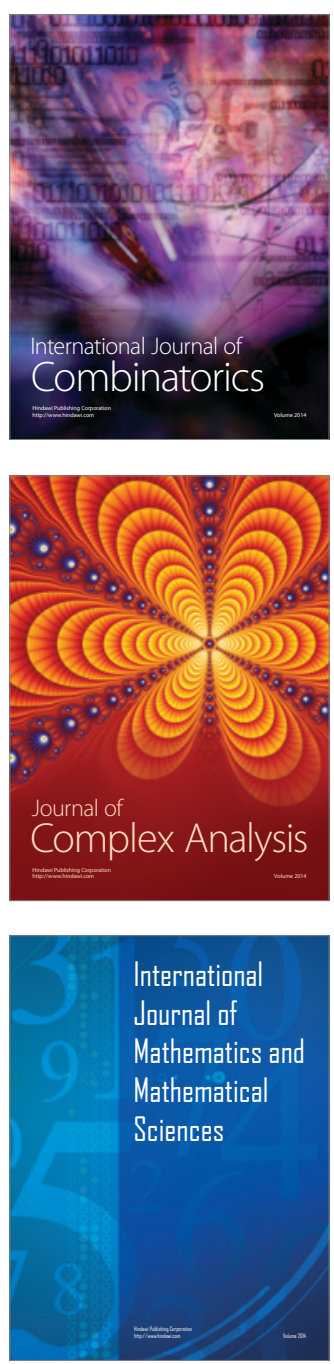
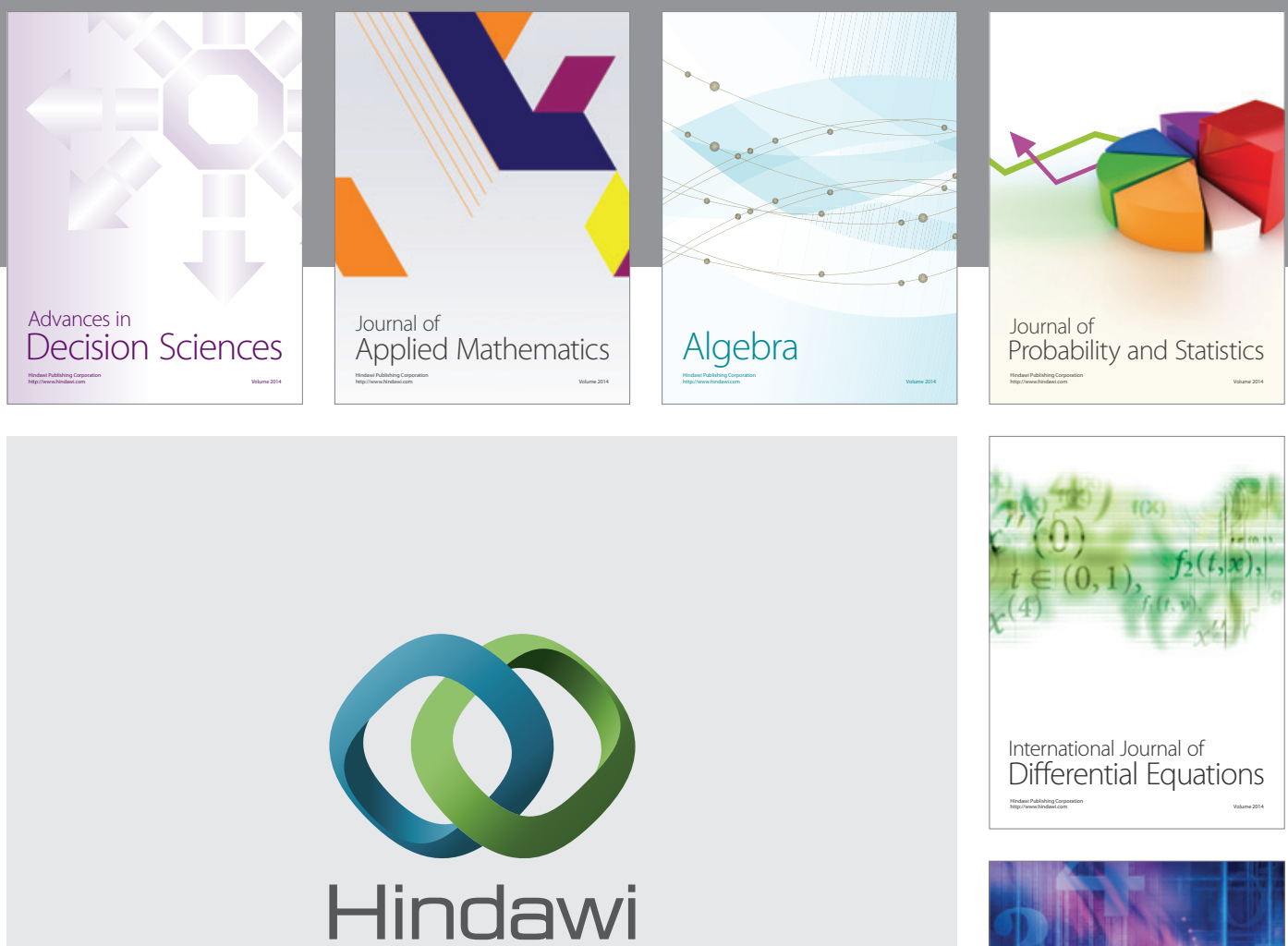

Submit your manuscripts at http://www.hindawi.com
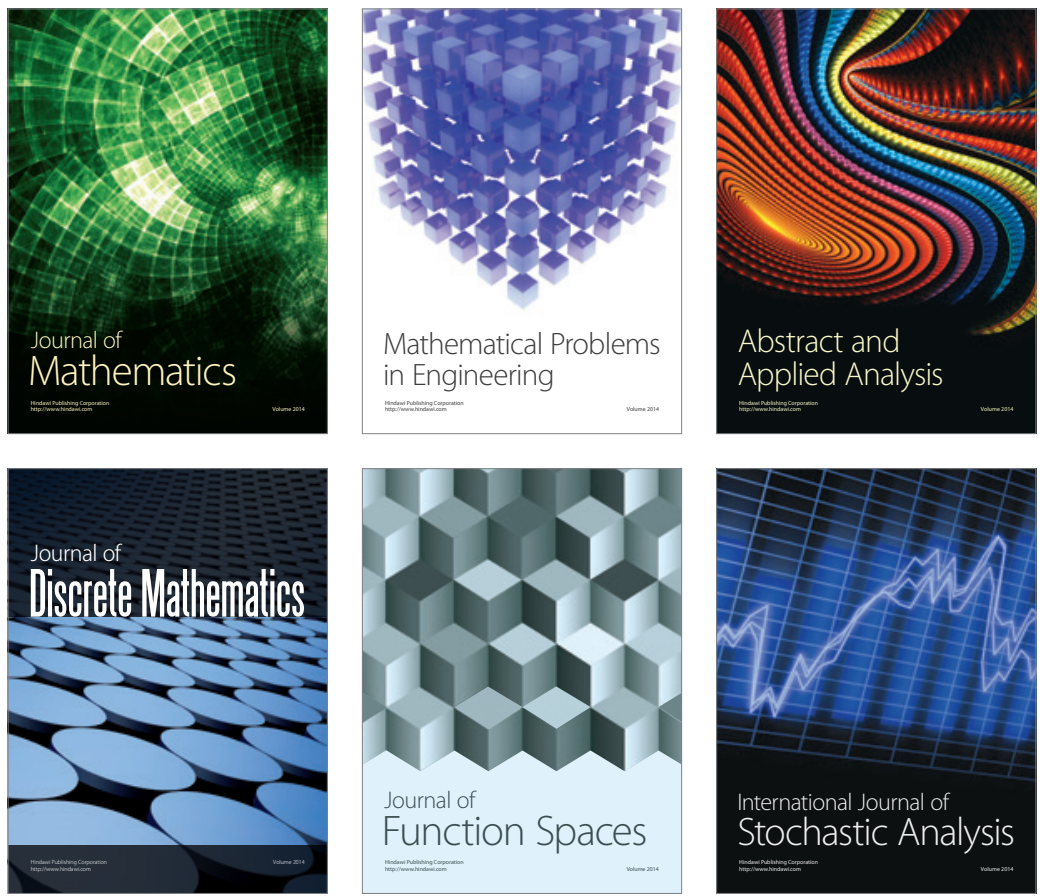

Journal of

Function Spaces

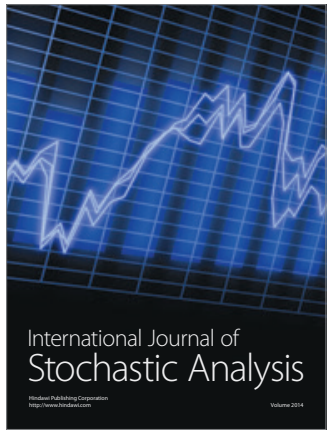

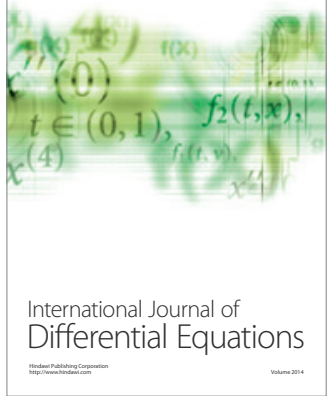
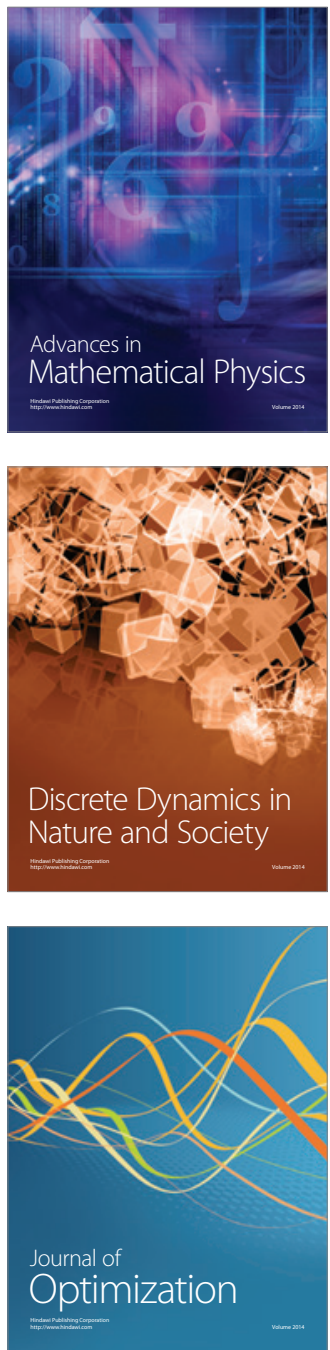